\title{
Avaliando a Formação de Professores no Contexto do Pensamento Computacional
}

\author{
Taiser T. T. Barros ${ }^{1,2}$, Eliseo Berni Reategui ${ }^{1}$, Ricardo Radaelli Meira ${ }^{3}$, \\ Adriano Canabarro Teixeira ${ }^{4}$
}

${ }^{1}$ PPGIE - Programa de Pós-Graduação em Informática na Educação

Universidade Federal do Rio Grande do Sul (UFRGS)

Av. Paulo Gama, 110 Anexo III - $3^{\circ}$ andar - Porto Alegre/RS CEP: 90040060

${ }^{2}$ EEP SENAI Carlos Tannhauser

Av. Gaspar Bartholomay 350, Santa Cruz do Sul/RS CEP: 96850000

${ }^{3}$ Instituto Federal Farroupilha

Unidade Cruz Alta - Cruz Alta, RS - Brazil

${ }^{4} \mathrm{PPG}$ em Educação/PPGEdu

Universidade de Passo Fundo - Passo Fundo, RS - Brazil

\{tttbarros78, eliseoreategui,ricardoradaelli\}@gmail.com, teixeira@upf.br

Resumo. Este artigo descreve a implementação de um programa de formação de professores oferecida à rede municipal de ensino de um município do estado do Rio Grande do Sul. Um total de 49 professores participaram da formação que trouxe conceitos relacionados ao Pensamento Computacional utilizando como ferramenta de implementação o Scratch. Uma análise do desempenho dos professores na utilização do Scratch dentro das 7 dimensões do Pensamento Computacional foi realizada utilizando a ferramenta Dr. Scratch. As impressões dos professores com relação à formação foram analisadas através das análises de conteúdo com processo de codificação. Os resultados obtidos sugerem determinada resistência por parte dos professores em integrar atividades relacionadas ao pensamento computacional em seus encargos e práticas de ensino correntes.

\begin{abstract}
This article describes a training program offered to a public education network in the state of Rio Grande do Sul, Brazil to train teachers to work with computational thinking. A total of 49 teachers participated in the program, which proposed the use of the tool Scratch. An analysis of the performance of teachers in the use of Scratch within the 7 dimensions of Computational Thinking was performed using Dr. Scratch. Teachers' perceptions regarding the training program were analyzed through content analysis with coding process. Results suggest a certain resistance from teachers to integrate computational thinking to their current tasks and teaching practices.
\end{abstract}

\section{Introdução}

O desenvolvimento de atividades do Pensamento Computacional (PC) em sala de aula é uma realidade em diversos países e instituições (BRACKMANN, 2017; MORAIS; 
BASSO; FAGUNDES, 2017). A partir da literatura na área, é possível identificar diferentes modelos para implementação de práticas para desenvolvimento do PC nas escolas, desde a criação de disciplinas específicas para isso, até a realização de atividades extra-curriculares. No Brasil, tais discussões têm-se intensificado principalmente com a inclusão do PC em diretrizes da Base Nacional Comum Curricular. Na prática, contudo, não está claro como o pensamento coputacional pode ser integrado ao currículo escolar: em que disciplina(s)? Qual a carga horária? Quais conteúdos? Que tipo de formação complementar deveria ser oferecida para os professores envolvidos?

Este artigo busca elucidar algumas destas dúvidas. No que diz respeito às áreas de formação dos professores que deveriam trabalhar nas escolas com o PC, a literatura aponta que a informática e a matemática são as áreas de formação dos professores que mais têmse envolvido com o tema. Neste contexto, no entanto, é importante destacar os relatos da resistência dos professores, de um modo geral, quanto ao uso de tecnologias, seja na utilização do computador ou na disponibilidade de aprender sobre uma linguagem de programação ou até mesmo outros tópicos relacionados à informática (TALLVID, 2016).

Com o objetivo de compreender como o ensino e aprendizagem do PC pode ser implementado nas escolas no contexto do Brasil, esta pesquisa apresenta um estudo realizado com 53 professores de matemática e de informática no sul do país que realizaram uma formação de 6 meses sobre o PC. As atividades de aprendizagem destes professores foram acompanhadas durante todo o período tanto para avaliá-las com relação às 7 dimensões do PC (MORENO-LEON; ROBLES; ROMAN-GONZALEZ, 2017), quanto para conhecer a percepção destes professores sobre como o PC pode ser integrado a suas práticas de sala de aula.

\section{O PC}

O PC pode ser compreendido como o "conjunto de atitudes e habilidades universalmente aplicáveis, que todos, não apenas cientistas da computação, deveriam estar ansiosos para aprender e usar" (WING, 2006). Já Blikstein (2008) define estas habilidades como: "Saber usar o computador como instrumento de aumento do poder cognitivo e operacional humano", sendo esta possivelmente a habilidade mais importante a ser desenvolvida por aqueles que necessitem estar aptos a exercer plenamente a cidadania no século XXI.

Introduzir o PC desde séries iniciais até o ensino médio é necessidade nas disciplinas de Matemática e Ciências dada a crescente incorporação de aspectos computacionais nestas áreas (WEINTROP et al., 2016). Tal tendência se mostra de acordo com Yadav, Stephenson e Hong (2017) que destacam a importância de os professores incorporarem o PC nas séries iniciais e no ensino médio, desde que tenham formação adequada. No Brasil a discussão sobre o PC e sua aplicação em sala de aula tem fomentado muitas discussões. Bombasar et al. (2015), identifica ferramentas utilizadas no ensino do PC e Araujo, Andrade e Guerrero (2016) apresentam uma mapeamento sistemático das abordagens, estímulos e instrumentos utilizados para avaliar progressos.

Destaca-se também que desde 2015, ocorre no país o WAlgProg ${ }^{1}$ com objetivo de promover a discussão e integração de pesquisas sobre métodos, técnicas e ferramentas que auxiliem no processo de ensino e aprendizagem do PC, Algoritmos e Programação.

\footnotetext{
${ }^{1}\langle$ http://walgprog.gp.utfpr.edu.br/> acesso em 21/10/2018.
} 
Entre as linguagens de programação e ferramentas, destaca-se a utilização do Scratch ${ }^{2}$, desenvolvido pelo grupo Lifelong Kindergarten do Massachusetts Institute of Technology (MIT) e amplamente utilizada para apoio ao desenvolvimento do PC. Trata-se de um ambiente de programação no qual é possível criar histórias interativas, jogos e animações. Algumas pesquisas mostram, por exemplo, como o Scratch pode ser utilizado para a aprendizagem de conceitos de programação e assim, em paralelo, impacta na aprendizagem matemática (CALAO et al., 2015) (ROCHA, 2015).

\subsection{Formação de Professores no contexto do PC}

No contexto do PC, Imberman, Sturm e Azhar (2014) destacam que a maior parte da formação oferecida aos professores se concentra em incentivar a incorporação do PC no currículo relacionado à informática e à matemática. Há também referências ao PC nas ciências, artes, história e biologia. Yadav et al. (2014) cita os professores do K-12 ${ }^{3}$ e como deve ser sua capacitação para que possam introduzir conceitos do PC em suas aulas. Porém, nestes estudos as iniciativas apresentadas ainda se limitam a professores da ciência da computação.

No Brasil, Valente (2016) destaca que a formação dos professores com relação ao PC é um grande desafio na medida em que não há consenso das áreas da tecnologia na educação e da ciência da computação sobre em que consiste o PC. Assim, é difícil estipular como abordar o tema na educação e, consequentemente, como formar educadores no assunto. Mesmo assim, diferentes iniciativas têm sido realizadas. França et al. (2014), por exemplo, apresentam um estudo sobre ações desenvolvidas pelo curso de Licenciatura em Computação da Universidade de Pernambuco para ensino dos fundamentos da Computação nas escolas. Rocha e Prado (2014) apresentam uma análise qualitativa sobre a formação continuada para professores de matemática utilizando o Scratch na qual eles são incentivados a criarem suas próprias ferramentas dentro da perspectiva construcionista observando que o software educativo é capaz de provocar reflexões do professor sobre sua prática em sala de aula. Contudo, como enfatizado por França e Tedesco (2015), é importante observar que a maior parte da literatura nacional sobre o PC traz relatos de estudos oriundos de projetos de pesquisa ou de extensão, sem considerar de maneira mais efetiva como a formação de professores ou o ensino do PC nas escolas pode ser implementado, efetivamente. Alinhados a esta visão, Silva, Silva e França (2017) destacam o desafio da formação de professores para trabalhar com o PC com relação à inadequação da infraestrutura nas escolas. A falta de planejamento para execução de cursos da área de computação na formação dos professores é outro ponto apontado pelos autores como problemático.

\section{Metodologia}

Esta pesquisa buscou contribuir com a discussão sobre a formação de professores para o ensino do PC nas escolas. Foi realizado um estudo de caso de caráter descritivo com abordagem de coleta e análise de dados quanti-qualitativa. O grupo participante da pesquisa foi consistuído por 49 professores do ensino fundamental, 12 da disciplina de Informática e 37 da disciplina de Matemática, da rede municipal de ensino de um município do estado do Rio Grande do Sul.

\footnotetext{
${ }^{2}$ Que foi utilizado na formação dos professores apresentada nesta pesquisa.

${ }^{3}$ Sistema de ensino público adotado em países como Estados Unidos da América (EUA), Canadá e Índia.
} 
Os referidos professores foram convidados, via Secretaria de Educação, a participar da formação cuja temática tratava sobre "o Desenvolvimento do PC utilizando o Scratch". Esta foi a ferramenta escolhida para a formação dada sua abrangência com relação às sete dimensões do PC (MORENO-LEON; ROBLES; ROMAN-GONZALEZ, 2017): abstração e decomposição de problemas, pensamento lógico, sincronização, paralelismo, noção algorítmica de controle de fluxo, interação com usuário e representação de dados. Outro motivo da escolha do Scratch foi em função de sua disponibilidade sem nenhum custo associado. Já o convite para participar da pesquisa foi enderaçado aos professores de informática das escolas, devido à relação direta com a temática da formação, bem como aos professores de matemática, área que também tem forte relação com a temática (WEINTROP et al., 2016) (BARCELOS; SILVEIRA, 2013). Além disto, as discussões relacionadas à implementação de um currículo envonvendo o PC na Educação Básica têm transitado mais frequentemente nestas duas áreas.

Com relação à formação, os professores foram divididos em duas turmas. Cada turma participou de 4 encontros presenciais de $4 \mathrm{~h}$ em um Centro de Formação Profissional que disponibilizou um laboratório de informática para as atividades. Os encontros ocorreram ao longo de um semestre letivo. No $1^{\circ}$ encontro foi apresentado o objetivo da pesquisa e os participantes assinaram um termo de Consentimento Livre e Esclarecido. Foram desenvolvidas atividades para trabalhar os comandos básicos do Scratch. No aguardo pelo segundo encontro, os professores tiveram apoio online (a distância) para elaboração de tarefas simples usando o Scratch com seus próprios alunos. Chegado o $2^{\circ}$ encontro, os professores fizeram o relato das atividades desenvolvidas utilizando os conceitos e comandos trabalhados na formação. Novos comandos e conceitos também foram trabalhados. No $3^{\circ}$ encontro, foram apresentadas as 7 dimensões do PC e foram discutidas diferentes maneiras de como implementar as mesmas empregando o Scratch. Neste momento, respeitadas as formações e atividades de cada professor (informática ou matemática), cada um foi solicitado desenvolver um projeto para trabalhar com conceitos do PC em sua própria sala de aula. No $4^{\circ}$ encontro realizou-se uma discussão sobre os projetos desenvolvidos como proposta de desenvolvimento do PC utilizando o Scratch. Em seguida, foi realizada uma entrevista semi-estruturada com os professores, com coleta de dados que consistiu na gravação do áudio da interação do pesquisador com o grupo, com posterior transcrição e codificação de dados.

\subsection{Análise de conteúdo e Avaliação dos Projetos Scratch}

Para analisar as falas dos professores utilizou-se a análise de conteúdo a partir do processo de codificação proposto por Saldana (2009). Dois professores com experiência em pesquisa na área, de modo independente, identificaram códigos e categorias de temas tratados nas entrevistas e discussões. O nível de concordância entre os dois codificadores foi verificado por meio da ferramenta online 4 que calcula o coeficiente de Cohen's Kappa classificando os níveis de concordância de "Pequeno" (0.00) a "Quase Perfeito" (1.00) (STEMLER, 2001). A primeira análise de concordância entre os códigos definidos por cada codificador apresentou $28.13 \%$ dos valores de coeficiente com classificação acima de "Moderado" (0.41 - 0.60). Após processo de conciliação proposto por Garrison et al. (2006), a totalidade da codificação atingiu classificação igual ou superior a "Significativa", validando assim o processo. Os códigos consolidados aparecem na figura 1.

\footnotetext{
${ }^{4}$ http://dfreelon.org/utils/recalfront/recal2/〉, acesso em 01/06/2018.
} 


\begin{tabular}{|c|c|c|}
\hline Categoria & Código & Descrição \\
\hline \multirow{3}{*}{ DIRETRIZES } & GRADE & Grade curricular da escola e/ou currículo implementado. \\
\hline & CNTDS & Para cumprir os conteúdos obrigatórios, não há tempo disponível para trabalhar o Scratch. \\
\hline & LEGIS & Falta documentação/ legislação que oriente a implementação da informática nas escolas. \\
\hline \multirow{3}{*}{ HABILIDADES } & HABAL & Habilidade dos alunos no contexto da informática: facilidade de programar, lógica, etc. \\
\hline & HABIP & Professor referenciou precisar de habilidades no Scratch para aplicar com os alunos. \\
\hline & DIFIC & Comentários dos professores sobre sua dificuldade com relação à informática. \\
\hline \multirow{2}{*}{\begin{tabular}{|l|} 
INTEGRAÇÃO \\
CURRICULAR \\
\end{tabular}} & INTER & Qualquer referência à interdisciplinaridade. \\
\hline & IMPLE & Como implementar o Scratch: jogos, projetos, oficina, etc. \\
\hline \multirow{5}{*}{$\begin{array}{l}\text { REAÇÃO DOS } \\
\text { PARTICIPANTES }\end{array}$} & ACETA & Aceitação dos alunos com relação ao Scratch. \\
\hline & ACETP & Aceitação dos professores com relação ao Scratch. \\
\hline & REJEA & Rejeição dos alunos com relação ao Scratch. \\
\hline & REJEP & Rejeição dos professores com relação ao Scratch. \\
\hline & RESPP & Professor reconhece que deve estudar para compreender como utilizar o Scratch. \\
\hline \multirow{6}{*}{$\begin{array}{l}\text { RECURSOS } \\
\text { DISPONIVEIS }\end{array}$} & PRFAX & Necessidade de um profissional que auxilie com questões de informática na escola. \\
\hline & ADEQO & Infraestrutura da escola está adequada para desenvolvimento de atividades. \\
\hline & DEFIC & Infraestrutura da escola não está adequada para desenvolvimento de atividades. \\
\hline & HORAP & Carga horária disponivel dos professores para trabalhar com informática. \\
\hline & HORAL & Horários disponíveis para utilizar os laboratórios de informática da escola. \\
\hline & NUMAL & Referência ao número de alunos em uma turma. \\
\hline
\end{tabular}

Figura 1. Categorias e Códigos.

A figura 2 mostra o número de ocorrências de cada código (correspondente à quantidade de professores que teve a fala classificada deste modo) que aponta para posicionamentos frequentes dos professores, como exemplo as 20 ocorrências do código ACETP, apontando para uma boa aceitação da ferramenta Scratch.

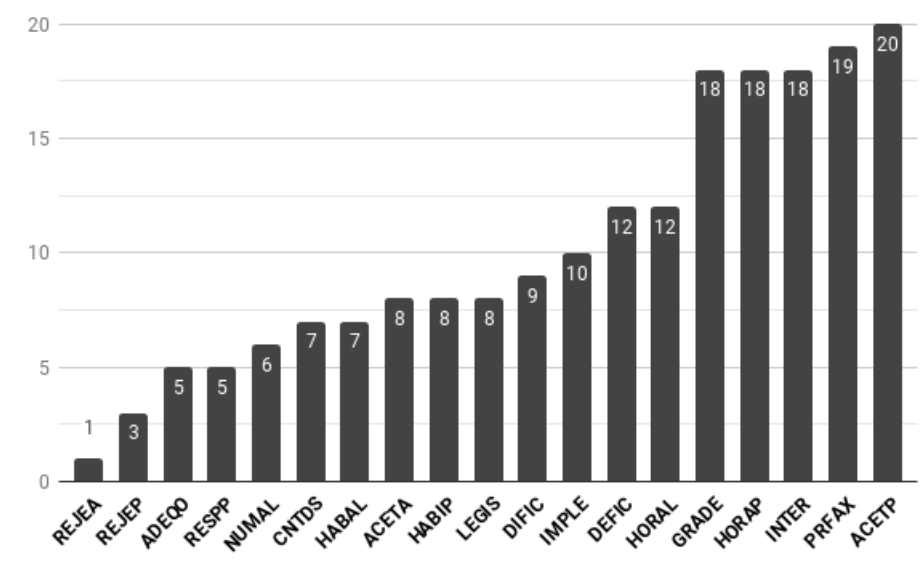

Figura 2. Quantidade de Ocorrências dos Códigos.

Já as 19 ocorrências do código PRFAX (79\% matemática) demonstram que estes professores não estão dispostos a assumir novas atividades para trabalhar com o PC sem o auxílio de um professor de informática. Este comportamento parece característico e aparece em estudos anteriores, como em Oliveira (2009) que aponta que professores menos ambientados com tecnologia apresentam menor interesse e disponibilidade em aprender sozinhos. Neste caso, o autor enfatiza que este comportamento pode estar relacionado ao currículo proposto no município onde a pesquisa foi realizada. Analogamente, Leite et al. (2017) enfatiza a demanda das escolas municipais de Francisco Beltrão no Paraná que imputam somente aos professores oriundos da Licenciatura em Informática a responsabilidade de trabalhar assuntos relativos ao PC.

Para verificar se problemas de infraestrutura teriam afetado a aceitação ao Scratch, relacionou-se o número de ocorrências do codigo REJEP com o do código DEFIC, sendo 
encontrado somente 1 ocorrência (matemática): "Desmotivada pois falta laboratório". Esta única ocorrência de fala sobre problemas de infraestrutura revela que esta dificuldade estava relativamente bem resolvida. Isso pode ser confirmado pelo número de ocorrências do códigos ADEQO, o qual classifica a infraestrutura das escolas no nível "Adequado" (NETO et al., 2013). Porém, mesmo com infraestrutura adequada, foi possível observar resistência à formação oferecida. Ma, Andersson e Streith (2005) enfatizam problema análogo, afirmando que somente a disponibilidade de infraestrutura de informática não é suficiente para garantir que o computador seja adotado como ferramenta de uso nas escolas.

A resistência dos professores de matemática também pode ser percebida analisando-se que dos 49 professores que participaram da pesquisa, apenas $26(70.27 \%)$ professores da matemática entregaram seu projetos, contra 12 de informática (100\%). Além disso, 8 de 9 ocorrências do código DIFIC aparecem nas falas da matemática, evidenciando um discurso que coloca a dificuldade com o uso da informática como um impeditivo para o desenvolvimento das atividades propostas. Contudo, na prática, foi possível observar que muitos destes professores não apresentaram reais dificuldades no uso da tecnologia. Mesmo assim, parecem ter necessidade de afirmar a mesma, similarmente ao que apresentou Curzon et al. (2009) durante o desenvolvimento de atividades de computação desplugada.

A ideia de que professores se preocupam com a necessidade de dominar determinado tópico é comprovada na análise das 8 ocorrências do código HABIP, como na fala de P08: "Preciso ter mais segurança (no uso do Scratch) para usar com meus alunos". Leite et al. (2017) também destaca a possibilidade da resistência dos professores ao uso da tecnologia estar fundamentada no paradigma dos currículos tradicionalistas. Penteado et al. (2000, p. 11) também discutem a constatação sobre resistência dos professores à informática, sinalizando que alguns encaram com desconfiança a introdução de novas tecnologias na Educação.

A resistência dos professores de matemática à formação proposta pode ser problemática se considerarmos que todas as menções ao PC no texto da Base Nacional Comum Curricular ${ }^{5}$ aparecem na área da matemática. Neste sentido, Morais, Basso e Fagundes (2017, p. 469) afirmam que é fundamental que o professor de matemática vislumbre a possibilidade de trabalhar com a aprendizagem de matemática enquanto ensina a seus estudantes princípios de programação.

A partir destas considerações é possível verificar que há expectativa com relação ao envolvimento dos professores de mátemática em atividades relacionadas ao pensamento computacional. Contudo, a realidade encontrada no estudo aqui apresentado mostrou-se um pouco diferente. A percepção da resistência por parte dos professores da matemática também fica evidente em alguns relatos, como na fala da professora de informática P37: "Faço um trabalho interdisciplinar mas não estou tendo apoio das professoras de matemática". "Me vejo pouco motivada no sentido da interdisciplinaridade, pois as professoras de matemática da escola não participam ativamente.". O que é contraditório, neste sentido, é que o código INTER, relativo à interdisciplinaridade, foi o terceiro em número de ocorrências nas falas dos professores, indicando a importância do

${ }^{5}$ BNCC, disponível em 〈http://basenacionalcomum.mec.gov.br//, acesso em 12/06/2018. 
tópico na percepção do grupo.

Outro aspecto relativo à forma como os professores de matemática reagiram à formação, observou-se uma única ocorrência coincidente dos códigos DIFIC e RESPP (no discurso de P04) ainda sendo que a ocorrência de RESPP para P04 se relaciona com a sua percepção de ter que P04: "Contextualizar a informática na vida dos alunos" e não com relação a se dedicar para sanar suas dificuldades ao contrário do que foi citado por P08: "Tenho que estudar nesta área (Scratch/informática)" e P30: "É mais a minha parte agora de estudar".

Deve-se destacar também a ocorrência de 8 considerações de aceitação por parte dos alunos, na visão dos professores (codigo: ACETA, 62.5\%), em relação a somente uma rejeição (codigo: REJEA) ao Scratch. O caso de rejeição foi específico a uma situação em que os alunos não tinham acesso ao computador em casa, corroborando fatores que afetam a utilização da tecnologia computacional (MA; ANDERSSON; STREITH, 2005). Pode-se concluir que os professores que tentaram utilizar o Scratch em aula tiveram bons resultados, similarmente ao que foi apontado por (MIOTTO; CARDOSO, 2014) que ensinou o conceito de função, posteriormente relatando interesse dos alunos nas atividades. Reforça-se também que os professores reconhecem nos alunos a capacidade de utilizar a tecnologia (frequência do código HABAL). Algumas das falas dos professores exemplificam estes conceitos: "Vão acabar ensinando o professor" e P48: "Eles (alunos) aprendem com muito mais facilidade que a gente (professores)".

As ocorrências do código IMPLE demonstram que, mesmo insistindo na dificuldade que possuem em informática, os professores (sejam da informática ou matemática) que buscaram utilizar o Scratch conseguiram de alguma forma implementar as atividades propostas. Realizaram oficinas, mostra de trabalhos ou projetos, assim como ilustra o discurso do professor P21: "a gente está desenvolvendo como trabalho com os alunos na forma de um projeto ... não teria como trabalhar com todos então a gente deu uma selecionada ... já no segundo encontro já surpreenderam como eles têm esta habilidade de tentar mexer no computador". Por fim, a ocorrência dos códigos NUMAL, CNTDS, LEGIS, HORAL, GRADE e HORAP expõe a necessidade da promoção de ambiente favorável à implementação do PC nas escolas. Estes códigos pertencentes às categorias DIRETRIZES e RECURSOS DISPONÍVEIS estão diretamente relacionados com o discurso dos professores em relação a itens que dependem da decisão de níveis hierárquicos superiores, como o da direção das escolas e secretarias de educação (municipais, estaduais). Os professores apontam a necessidade destas instituições revisarem conteúdos, carga horária e disponibilidade de infraestrutura para que o PC possa ser trabalhado de maneira sistemática.

Duas das atividades de avaliação realizadas na formação foram consideradas na pesquisa. A primeira delas, por meio do desenvolvimento de um programa simples, buscou avaliar o que os professores aprenderam a partir do seu primeiro contato com o Scratch. A segunda atividade solicitava aos professores que fizessem um programa no Scratch acompanhado de prática pedagógica para apoio à aprendizagem do pensamento computacional. Os programas desenvolvidos pelos docentes foram avaliados utilizando a ferramenta $D r$. Scratch ${ }^{6}$, que atribui uma pontuações de 0 a 21 pontos levando em

\footnotetext{
${ }^{6}\langle$ http://www.drscratch.org/ $\rangle$, acesso em 01/06/2018
} 
consideração as 7 dimensões do pensamento computacional.

O cálculo da média das notas para a primeira atividade foi de 7,02 e 8,78 para a segunda atividade, evidenciando uma pequena evolução no desempenho dos alunos ao longo da formação. Mesmo assim, o número fica bem distante da pontuação máxima de 21 pontos. No entanto, cabe aqui destacar a diferença de desempenho observada entre os professores de matemática e os professores de informática: enquanto a média do primeiro grupo alcançou o valor de 6,25, a média do segundo grupo atingiu o valor de 8,10 , valor um pouco superior com p-value $=0,037$ computado pelo teste-t de Student, evidenciando diferença significativa. Apesar da diferença das médias não ser muito alta, cabe mesmo assim observar-se as limitações de uma formação mais pontual, como esta oferecida, para capacitar os professores no desenvolvimento das diversas dimensões do pensamento computacional. Os professores de matemática foram capazes de desenvolver proposta mais simples, envolvendo conceitos mais elementares do pensamento computacional. Já os professores de informática conseguiram ir um pouco além, construindo programas e trazendo propostas mais complexas.

A resistência dos professores à formação oferecida também pôde ser observada na entrega de atividades. Muitos professores entregaram o menor número de atividades possível para obterem a certificação do curso, o que foi observado tanto pelo registro de atividades mantido pelo formador quanto por comentários feitos no grupo do Whatsapp do qual o formador fazia parte.

\section{Conclusões}

Este trabalho apresentou uma proposta de formação de professores no contexto do PC. Projeto Scratch foram desenvolvidos e analisados com relação às 7 dimensões do PC mostrando que pelo menos o nível básico conforme o Dr. Scratch foi atingido. As análises de conteúdo e de discurso juntamente com a codificação, permitiram avaliar as impressões dos professores com relação à formação proposta.

Observou-se que existe certa resistência dos professores, principalmente do grupo da matemática, em aceitar o PC como ferramenta de sala de aula, sendo este resultado similar aos encontrados na literatura, assim como a citação à Penteado et al. (2000) na seção 3.1, que constata sobre a resistência à informática em um trabalho publicado há 18 anos, mostrando que o processo de aceitação da tecnologia pode ser lento em relação ao avanço da mesma.

Ainda, a resistência por parte dos professores é problemática uma vez que o apoio dos mesmos é fundamental para que se possa disseminar os conceitos do PC. Como trabalhos futuros, pretende-se realizar visitas nas escolas onde os participantes da pesquisa atuam, verificando quais as condições de trabalho e infraestrutura que possuem, buscando estratégias que aumentem a aceitação à utilização do PC.

\section{Referências}

ARAUJO, A. L. S. O. de; ANDRADE, W. L.; GUERRERO, D. D. S. Um mapeamento sistemático sobre a avaliação do pensamento computacional no brasil. In: Anais dos Workshops do Congresso Brasileiro de Informática na Educação (CBIE 2016). [S.1.]: SBC - Sociedade Brasileira de Computação, 2016. p. 1147-1158. 
BARCELOS, T. S.; SILVEIRA, I. F. Relações entre o pensamento computacional e a matemática através da construção de jogos digitais. In: Proceedings of SBGames 2013. [S.1.]: SBC - Sociedade Brasileira de Computação, 2013. p. 52-55.

BLIKSTEIN, P. O pensamento computacional e a reinvenção do computador na educação. 2008. Publicação online. Disponível em: 〈http://www.blikstein.com/paulo/ documents/online/ol \_pensamento $\backslash$ _computacional.html $\rangle$.

BOMBASAR, J. et al. Ferramentas para o ensino-aprendizagem do pensamento computacional: onde está alan turing? In: COMPUTAçãO, S. S. B. de (Ed.). Anais do XXVI Simpósio Brasileiro de Informática na Educação (SBIE 2015). [S.1.]: SBC Sociedade Brasileira de Computação, 2015. p. 81-90. ISSN 2316-6533.

BRACKMANN, C. P. Desenvolvimento do Pensamento Computacional Através de Atividades Desplugadas na Educação Básica. Tese (Doutorado) - Universidade Federal do Rio Grande do Sul (UFRGS), Porto Alegre - RS, 2017.

CALAO, L. A. et al. Developing mathematical thinking with scratch. In: Design for Teaching and Learning in a Networked World: 10th European Conference on Technology Enhanced Learning, EC-TEL 2015, Toledo, Spain, September 15-18, 2015, Proceedings. Cham: Springer International Publishing, 2015. p. 17-27.

CURZON, P. et al. Enthusing \& inspiring with reusable kinaesthetic activities. SIGCSE Bull., v. 41, n. 3, p. 94-98, jul. 2009. ISSN 0097-8418.

FRANçA, R.; TEDESCO, P. Desafios e oportunidades ao ensino do pensamento computacional na educação básica no brasil. In: Anais dos Workshops do IV Congresso Brasileiro de Informática na Educação (CBIE 2015). [S.1.]: SBC Sociedade Brasileira de Computação, 2015. p. 1464-1473.

FRANçA, R. S. de et al. A disseminação do pensamento computacional na educação básica: lições aprendidas com experiências de licenciandos em computação. In: Anais do XXXIV Congresso da Sociedade Brasileira de Computação (CSBC 2014). [S.1.]: SBC - Sociedade Brasileira de Computação, 2014. p. 1505-1514.

GARRISON, D. et al. Revisiting methodological issues in transcript analysis: Negotiated coding and reliability. The Internet and Higher Education, v. 9, n. 1, p. 1-8, 2006. ISSN 1096-7516.

IMBERMAN, S.; STURM, D.; AZHAR, M. Q. Computational thinking: expanding the toolkit. v. 29, p. 39-46, 062014.

LEITE, M. et al. Pensamento computacional nas escolas: Limitado pela tecnologia, infraestrutura ou prática docente? In: Anais dos Workshops do VI Congresso Brasileiro de Informática na Educação (CBIE 2017). [S.1.]: SBC - Sociedade Brasileira de Computação, 2017. p. 1002-1010.

MA, W. W.-k.; ANDERSSON, R.; STREITH, K.-O. Examining user acceptance of computer technology: an empirical study of student teachers. Journal of Computer Assisted Learning, Blackwell Publishing Ltd, v. 21, n. 6, p. 387-395, 2005.

MIOTTO, P.; CARDOSO, V. C. A utilização do software scratch para o ensino e a aprendizagem do conceito de função. Os Desafios da Escola Pública Paranaense na Perspectiva do Professor PDE, I, 2014. 
MORAIS, A. D. d.; BASSO, M. V. d. A.; FAGUNDES, L. A. d. C. Educação Matemática Ciência da Computação na escola: aprender a programar fomenta a aprendizagem de matemática? Ciência Educação (Bauru), scielo, v. 23, p. 455 - 473, 062017.

MORENO-LEON, J.; ROBLES, G.; ROMAN-GONZALEZ, M. Towards data-driven learning paths to develop computational thinking with scratch. IEEE Transactions on Energing Topics in Computing, v. 16, Aug 2017. ISSN 2168-6750.

NETO, J. J. S. et al. Uma escala para medir a infraestrutura escolar. Estudos em Avaliação Educacional, v. 24, n. 54, p. 78 - 99, jan 2013.

OLIVEIRA, E. C. de L. O uso do Scratch no ensino fundamental: possibilidades de incorporação curricular segundo professoras dos anos iniciais. Dissertação (Mestrado) - Pontifícia Universidade Católica de Minas Gerais, Belo Horizonte, 2009.

PENTEADO, M. et al. A informática em ação: formação de professores, pesquisa e extensão. $1^{\text {a }}$. ed. São Paulo, SP: Olho d'Água, 2000.

ROCHA, A. K. d. O.; PRADO, M. E. B. B. Uma abordagem tecnológica na formação do professor de matemática. Revista Tecnologias na Educação, Ano 6, n. 11, 2014.

ROCHA, K. C. da. Programando com o scratch na aula de matemÁtica. Revista RENOTE - Novas Tecnologias na Educação, v. 13, n. 2, p. 1-10, mar. 2015.

SALDANA, J. The Coding Manual for Qualitative Researchers. SAGE Publications, 2009. ISBN 9781446200124. Disponível em: 〈https://books.google.com.br/books?id= msuOE0UfXpUC $\rangle$.

SILVA, V.; SILVA, K.; FRANçA, R. S. de. Pensamento computacional na formação de professores: experiências e desafios encontrados no ensino da computação em escolas públicas. In: Anais do XXIII Workshop de Informática na Escola (WIE 2017) do IV Congresso Brasileiro de Informática na Educação (CBIE 2017). [S.1.]: SBC Sociedade Brasileira de Computação, 2017. p. 805-814.

STEMLER, S. An overview of content analysis. Practical Assessment, Research \& Evaluation, v. 7, n. 17, Jun 2001.

TALLVID, M. Understanding teachers' reluctance to the pedagogical use of ict in the 1: 1 classroom. Education and Information Technologies, v. 21, n. 3, p. 503-519, maio 2016. ISSN 1360-2357.

VALENTE, J. Integração do pensamento computacional no currículo da educação básica: diferentes estratégias usadas e questões de formação de professores e avaliação do aluno. Revista e-Curriculum, v. 14, n. 3, p. 864-897, 2016. ISSN 1809-3876.

WEINTROP, D. et al. Defining computational thinking for mathematics and science classrooms. Journal of Science Education and Technology, Springer Netherlands, v. 25, n. 1, p. 127-147, 2 2016. ISSN 1059-0145.

WING, J. M. Computational thinking. Commun. ACM, v. 49, n. 3, p. 33-35, mar. 2006.

YADAV, A. et al. Computational thinking in elementary and secondary teacher education. v. 14, 032014.

YADAV, A.; STEPHENSON, C.; HONG, H. Computational thinking for teacher education. Commun. ACM, v. 60, n. 4, p. 55-62, mar. 2017. ISSN 0001-0782. 